\title{
A SIMPLE ANALYSIS OF DIELECTRIC PROPERTIES OF PZT
}

\author{
Alokesh Mondal ${ }^{1}$, Biswajit Maiti² and Achintya Das ${ }^{3}$ \\ ${ }^{1}$ Department of Electronics and Communication Engineering, Durgapur Institute of Advanced Technology \\ and Management, Rajbandh, Durgapur, Burdwan - 713212, India. Email : alokesh82@yahoo.co.in \\ 2Department of Physics, Kalyani Government Engineering College, Kalyani, Nadia - 741235, India. \\ Email : bmkgec@gmail.com \\ 3Department of Electronics and Communication Engineering, Kalyani Government Engineering College, \\ Kalyani, Nadia - 741235, India. Email : achintya.das123@gmail.com
}

\begin{abstract}
Lead Zirconate Titanate (PZT), a very important perovskite material, has been fabricated by ceramic processing method. Dependence of dielectric properties on temperature and frequency of electric field has been investigated. A two layered RC circuit model resembling grain and grain-boundary is employed to analyze the variation of dielectric constant with frequency of electric field. Fluctuations in the measure of dielectric constant are indicative of the presence of defects and impurities in the grain boundary region. Temperature effect has been described by the structural change caused by phase transition which is quite susceptible to the ratio of $\mathrm{Zr}$ to $\mathrm{Ti}$ in material composition. Specifically near morphotropic phase boundary (MPB) with the presence of mixed tetragonal and rhombohedral phases, a large variation of dielectric constant can effectively be explained by Maxwell-Wagner relaxation of polarization charges at the grain-grain boundary interface.
\end{abstract}

Keywords : Perovskite material, ceramic processing method, dielectric constant, morphotropic phase boundary, grain and grain-boundary, Maxwell-Wagner relaxation.

\section{Introduction}

In ceramic materials, electrical conduction generally involves diffusion of ionic and electronic charge carriers and the balance is attained by temperature and ambient condition. Electrical conduction is generally controlled by the presence of impurity atoms and the complication arises possibly due to overriding of grain boundary regions, i.e., depletion of charges into the grains and also due to the presence of other phases. In ceramic materials drift of charge carriers under applied electric field is forbidden due to space charge polarization at the graingrain boundary interfaces [1]. Only a small fraction of charges can move upto grain boundary region. This effect can be modelled effectively by a two layered $\mathrm{RC}$ equivalent

equivalent electrical circuit. In a simple parallel $R C$ equivalent electrical circuit subjected to an alternating electric field, the output current delay can reach zero value as is explained by single relaxation time and the frequency response of impedance shows a semicircle due to lossy capacitor. In this model, grain-boundary is replaced by an equivalent lossy capacitor, while the grains are modelled as resistive elements [1]. When an electric current passes through such a material containing the interfaces between two different dielectric media with different conductivities, surface charges pile up at the interfaces and gives rise to Debye like relaxation under an alternating electric field [2-6]. 
It is found in a large number of articles that all the perovskite type materials like Lead Zirconate Titanate (PZT) show two electrical response peaks in impedance formalism and this could be interpreted, as modeled above, by a two-layered RC electrical circuit with partly conducting grains separated from each other by weakly conducting grain boundaries [2-3]. Effect of interfacial polarization occurring in these materials has effectively been explained by Larsen et al. using Maxwell-Wagner relaxation [3, 7-8].

The $\mathrm{Pb}\left(\mathrm{Zr}_{1-\mathrm{x}} \mathrm{Ti}_{\mathrm{x}}\right) \mathrm{O}_{3}(\mathrm{PZT})$ phase diagram is strongly composition-dependent and formed a highly symmetrical primitive cubic structure beyond Curie temperature $\left(T_{C}\right)$. At room temperature, the tetragonal phase exists for Ti-rich PZT and the rhombohedral phase exists for Zr-rich PZT. These two phases are ferroelectric in nature. Material properties, specifically dielectric properties, are strongly dependent on different phases caused by different composition ratio of $\mathrm{Zr}$ to $\mathrm{Ti}$. For near stoitiometric ratio of $\mathrm{Zr} / \mathrm{Ti}$ $(53 / 47)$ both the tetragonal and rhombohedral perovskite phases occur in a mixed phase state and is known as morphotropic phase boundary (MPB). The material properties are optimum at this region of morphotropic phase boundary (MPB). At MPB, dielectric constant is higher by several orders of magnitude in the polarization directions compared to a single phase composition [9]. Highest piezoelectric coefficient is found to occur at MPB and it corresponds to the peak of spontaneous polarization. An extrinsic piezoelectric contribution sharply increases due to degeneracy between the tetragonal and rhombohedral phases that leads to easy reorientation of ferroelectric domains under applied electric field or mechanical stress [10]. Though PZT is a widely studied material, but there is the scope of theoretical analysis of its properties through a simple $\mathrm{RC}$ equivalent circuit model which is the subject of this article.

For experimental verification of this equivalent circuit model, PZT pallets of different composition have been fabricated in this work near to MPB. In this case phase transition occurs from zirconium rich rhombohedral to titanium rich tetragonal phase resulting in high dielectric properties even at room temperature. The determination of permittivity as a function of temperature has shown almost constant value between room temperature to approximately $70^{\circ} \mathrm{C}$. This is indicative of good thermal stability of the material. Variation of dielectric constant as function of temperature and frequency has been found to have broadened curve around $T_{C}$ due to compositional fluctuations and/or substitutional disordering of the cation arrangement in the crystal structure [12-12]. This may be due to microscopic heterogeneity in the crystal with different local curie points. High value of the dielectric constant has also been found over a wide temperature range. Dielectric constant as a function of frequency has revealed an almost constant value at low frequency zone and exponential decrease towards higher frequencies. This decrease of dielectric constant may be determined from the study of loss peak in the imaginary part of the dielectric constant. It is seen to have an exponential shift towards higher frequencies with increasing temperature and has the Debye like dipolar relaxation characteristic [13-21]. 


\section{Experimental}

Ferroelectric PZT samples have been fabricated by the ceramic processing method using Lead dioxide, Zirconium dioxide and Titanium dioxide as starting materials. The purity of these materials was approximately $99 \%$. The raw materials were mixed and milled for two hours in planetary ball mill with zirconia balls of different sizes in an alumina crucible leading to finer particles of average particle size $\sim 1 \mu \mathrm{m}$. The powder mixture was dispersed in polyvinyl alcohol (PVA) and a little amount of Manhattan fish oil was used to get sustained dispersion and then it was placed in isostatic press at $400 \mathrm{kPa}$ pressure for four minutes for the preparation of green pallet. It was then calcined in steps followed by sintering at a temperature of $1400^{\circ} \mathrm{C}$. The as-prepared PZT disc was of $98.2 \%$ density and its thickness was $1.06 \mathrm{~mm}$, diameter was 2.15 $\mathrm{cm}$. The PZT sample compositions were taken as $45 \%$ Titanate and $55 \%$ Zirconate. The prepared ceramic sample was sandwiched between two gold plated brass plates of same diameter as the PZT sample to design a parallel plate capacitor for the purpose of experimentation.

\section{Results}

As the fabricated PZT material is compositionally little off from stoitiometry, the excess $\mathrm{Zr}^{4+}$ ions would attract neighbouring oxygen atoms more towards them compared to the $\mathrm{Ti}^{4+}$ and the resulting distortion or lattice deformation creates spontaneous polarization. The ionic radius of $\mathrm{Zr}$ being larger than that of Ti structural distortion also may occur. In a polycrystalline ceramic material like this one, grain and grain boundary region plays an important role in the dielectric behavior and transport properties as well. At higher temperatures some of the trapped charges at the grain boundaries or at the impurity sites may be relaxed to give it a little electrical conduction, through the process of hoping conduction. Also the observed large dielectric constant in these materials close to Tc is mainly due to the hopping of charge carriers $[3,4]$. The amount of spontaneous polarization will change due to this hopping. This is estimated from the study of ac conduction through the material. Fig. 1 shows the sample and the sample holder of the experimental set up.

To study these effects analytically, polycrystalline PZT sample is modeled as an electrical network by assigning separate resistance and capacitance to the grain and grain boundary regions and here they are assumed to have an even distribution throughout the sample. To analyze ac impedance, the grain-boundary region is modeled as parallel combination of resistance $R_{g b}$ and capacitance $C_{g b}$, while grains are taken as a resistive element of resistance $R_{g}$ and is shown in Fig.2.

$A C$ conduction was measured at room temperature in an L-C-R meter and the Nyquist plot between real part $\left(Z^{\prime}\right)$ and imaginary part $\left(Z^{\prime \prime}\right)$ of complex impedance $Z^{*}\left(=Z^{\prime}-j Z^{\prime \prime}\right)$ is plotted.

Fig.2 represents equivalent electrical circuit of a single block of grain and grain-boundary region of the material, the complex impedance of which is given by

$$
\begin{aligned}
Z^{*} & =R_{g}+\frac{R_{g b}}{1+j \omega R_{g b} C_{g b}} \\
& =\left(R_{g}+\frac{R_{g b}}{1+\omega^{2} R_{g b}{ }^{2} C_{g b}{ }^{2}}\right)-j \frac{\omega R_{g b}{ }^{2} C_{g b}}{1+\omega^{2} R_{g b}{ }^{2} C_{g b}{ }^{2}} \\
& =Z_{e q}^{\prime}-j Z_{e q}^{\prime \prime}
\end{aligned}
$$


where, real part of impedance is

$$
Z_{e q}^{\prime}=\left(R_{g}+\frac{R_{g b}}{1+\omega^{2} R_{g b}^{2} C_{g b}^{2}}\right)
$$

and imaginary part is

$$
Z_{e q}^{\prime \prime}=\frac{\omega R_{g b}{ }^{2} C_{g b}}{1+\omega^{2} R_{g b}{ }^{2} C_{g b}{ }^{2}}
$$

From Eq. (2) and Eq. (3) it is evident that the grain and grain boundary response peaks are located at infinity and at $\frac{1}{\left(2 \pi R_{g b} C_{g b}\right)}$, respectively. These values of response peaks are proportional to associated resistances and capacitance of grains and grain boundary regions [22]. In this equivalent circuit model, the resonance peak frequency of grain is at infinity due to zero grain capacitance. But, the peak value for grain boundary is finite. The resistance of grain boundaries being much higher than that of grains there is the possibility of charge polarization and hopping conduction between the grains. Defect or impurity may act as trap states in grain boundary regions leading to little electronic conduction at high temperatures due to charge relaxation from the trap states.

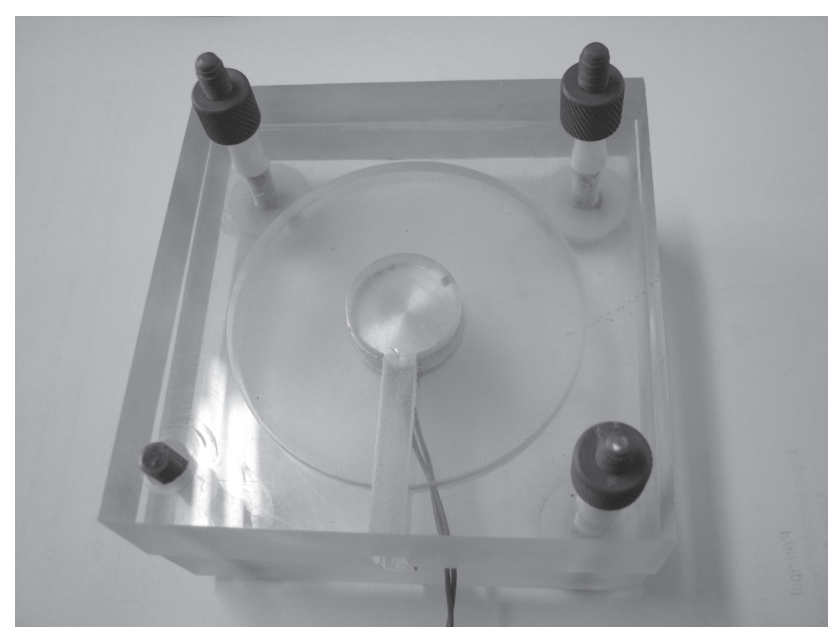

(a)

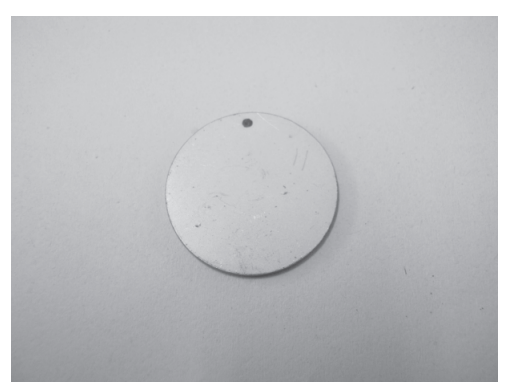

(b)

Fig.1 (a) The sample holder, and (b) the PZT sample

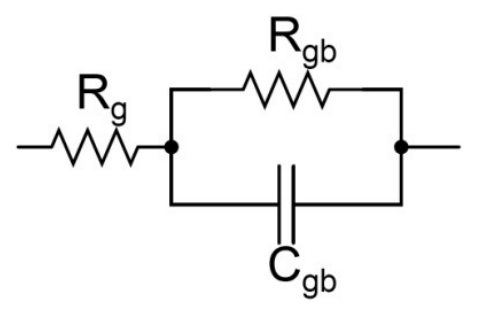

Fig.2 Equivalent electrical circuit used to represent the electrical properties of grain and grain-boundary

Assuming the resistance of grain $\left(R_{g}\right)=$ $6.066 \times 10^{9} \Omega$, resistance of grain boundary $\left(R_{g b}\right)=1.25524 \times 10^{12} \Omega$ and capacitance of grain boundary $\left(\mathrm{C}_{\mathrm{gb}}\right)=0.1321 \mathrm{pF}$ for the designed PZT pallet, the analytical results show best fit with the experimental findings.

The dielectric constant corresponding to the equivalent electrical circuit of one such block is represented by the following equation

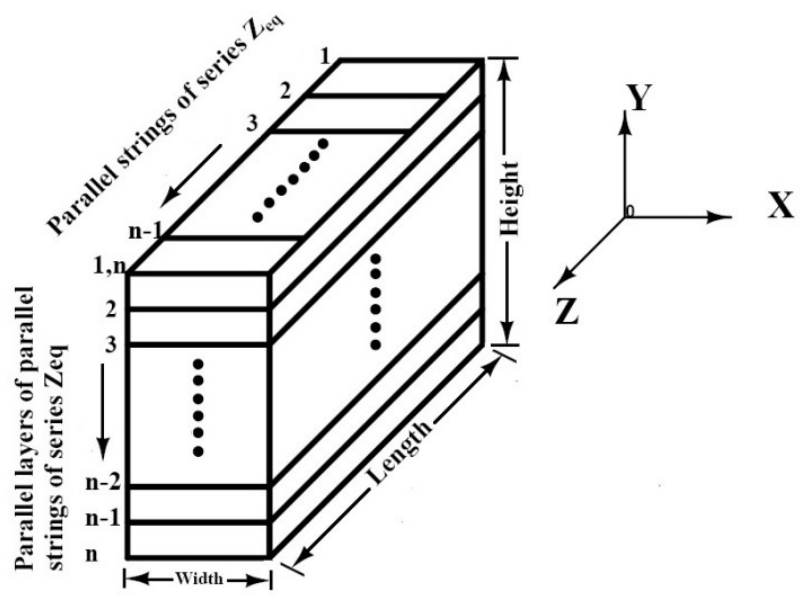

Fig.3 Cubic structure of PZT sample 


$$
\begin{gathered}
\varepsilon_{\text {eq }}^{*}=\varepsilon_{\text {eq }}^{\prime}-j \varepsilon_{\text {eq }}^{\prime \prime}=\frac{1}{j \omega C_{\text {oeq }} Z_{\text {eq }}^{*}} \\
=\frac{Z_{\text {eq }}^{\prime}}{\omega C_{\text {oeq }}\left(Z_{\text {eq }}^{\prime}+Z_{\text {eq }}^{\prime \prime}\right)}-j \frac{Z_{\text {eq }}^{\prime \prime}}{\omega C_{\text {oeq }}\left(Z_{\text {eq }}^{\prime}{ }^{2}+Z_{\text {eq }}^{\prime \prime}{ }^{2}\right)}
\end{gathered}
$$

The real part of dielectric constant is

$$
\varepsilon_{\text {eq }}^{\prime}=\frac{Z_{e q}^{\prime}}{\omega C_{\text {oeq }}\left(Z_{\text {eq }}^{\prime 2}+Z_{\text {eq }}^{\prime \prime 2}\right)}
$$

While the imaginary part of dielectric constant is

$$
\varepsilon_{\text {eq }}^{\prime \prime}=\frac{Z_{\text {eq }}^{\prime \prime}}{\omega C_{\text {oeq }}\left(Z_{\text {eq }}^{\prime 2}+Z_{\text {eq }}^{\prime \prime}\right)}
$$

The free space capacitance of the capacitor of such dimension is $C_{\text {oeq }}=14.1664$ $x 10^{-15} \mathrm{~F}$.

$106 \mathrm{~mm} A$-rectangular block of PZT pallet of

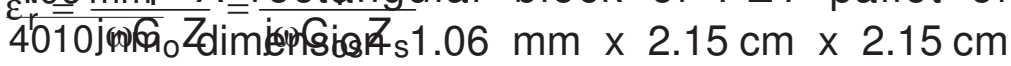
has $m$ number of impedance blocks having impedance Zeq in series in the $X$ direction forming a string of impedances and again $n$ number of such impedance strings with equivalent impedance $\mathrm{mZeq}$ in the $\mathrm{Z}$ direction, the combination shows $n$ number of such layers as parallel impedance plates in the $\mathrm{Y}$ direction (Fig. 3). Zs represents the total impedance of the PZT pallet as series parallel combination of impedance blocks in different layers. As the sample thickness is $\mathrm{t}=1.06 \mathrm{~mm}$ and thickness of a single block of grain and grain-boundary region is $d=$ $4 \mu \mathrm{m}$ (dimension of grain) $+10 \mathrm{~nm}$ (dimension of grain boundary) $=4.01 \mu \mathrm{m}$, the total number of impedance blocks in the $X$ direction will be $\mathrm{m}=\frac{\mathrm{t}}{\mathrm{d}^{\prime}}=\quad=265$ and it is taken as thickness factor.
The total number of strings in $Z$ direction is then $\mathrm{n}=\frac{\mathrm{L}}{\mathrm{d}^{\prime}}=\frac{2.1535314 \mathrm{~cm}}{4010 \mathrm{~nm}}=5370$.

The total number of layers of strings in $Y$ direction is

$$
\mathrm{n}=\frac{\mathrm{H}}{\mathrm{d}^{\prime}}=\frac{2.153514 \mathrm{~cm}}{4010 \mathrm{~nm}}=5370
$$

Therefore, the complex impedance of the $\mathrm{PZT}$ pallet is

$$
Z_{\mathrm{s}}=\frac{m Z_{e q}}{n^{2}}=9.189614695 \times 10^{-6}
$$

$\left[\left(R_{g}+\frac{R_{g b}}{1+\omega^{2} R_{g b}{ }^{2} C_{g b}{ }^{2}}\right)-j \frac{\omega R_{g b}{ }^{2} C_{g b}}{1+\omega^{2} R_{g b}{ }^{2} C_{g b}{ }^{2}}\right]$ (7)

So, the real part of impedance of the PZT pallet is

$$
\begin{array}{r}
Z_{s}^{\prime}=9.18961469506 \times 10^{-6} \\
\left(R_{g}+\frac{R_{g b}}{1+\omega^{2} R_{g b}^{2} C_{g b}^{2}}\right)
\end{array}
$$

and the imaginary part of impedance is

$$
Z_{s}^{\prime \prime}=9.18961469506 \times 10^{-6}\left(\frac{\omega R_{g t}{ }^{2} C_{g b}}{1+\omega^{2} R_{g b}{ }^{2} C_{g b}{ }^{2}}\right)
$$

The dielectric constant, $\varepsilon_{r}^{*}=\varepsilon_{r}^{\prime}-j \varepsilon_{r}^{\prime \prime}$, of the $\mathrm{PZT}$ pallet is given by

$$
=\frac{Z_{s}^{\prime}}{\omega C_{o s}\left(Z_{s}^{\prime 2}+Z_{s}^{\prime \prime 2}\right)}-j \frac{Z_{s}^{\prime \prime}}{\omega C_{o s}\left(Z_{s}^{\prime 2}+Z_{s}^{\prime \prime 2}\right)}
$$

The real part of which is

$$
\varepsilon_{r}^{\prime}=\frac{Z_{s}^{\prime}}{\omega C_{o s}\left(Z_{s}^{\prime 2}+Z_{s}^{\prime \prime 2}\right)}
$$

